\title{
Hotel Industry: Research on the Relationship between Team Culture, Organizational Learning and Service Innovation Zhang Chunhuan ${ }^{1, a}$
}

${ }^{1}$ College of economics and trade, South China University of Technology, Guangzhou, Guangdong, China

aemail,328536036@qq.com

${ }^{*}$ Corresponding author,Zhang Chunhuan

Keywords: Service Innovation; Team Culture; Organizational Learning

\begin{abstract}
In recent years, the rapid development of our hotel also ushered in more and more challenges, how to improve the occupancy rate, how will the decline in the momentum of the hotel to enhance the profits, how to meet the diverse needs of consumers, as various types of hotel management The primary question of thinking. Service innovation because of its quality of service to improve the hotel to improve customer satisfaction and hotel profits by the hotel managers and academic researchers attention.Based on the previous scholars on the service innovation research, from the perspective of team culture and organizational learning to explore its role in the hotel service innovation, mainly within the country several major cities in the high-end hotels, including the economy in recent years Hotels and themed hotels to analyze the existing hotel attention to the degree of service innovation and how to achieve service innovation, and then verify the impact of these hotel service innovation factors.
\end{abstract}

\section{酒店业：团队文化、组织学习与服务创新的关系研究}

\author{
张春焕 $1, \mathrm{a}$ \\ 1 华南理工大学经济与贸易学院, 广州, 广东, 中国 \\ aemail, 328536036@qq.com
}

*通讯作者, 张春焕

关键词: 服务创新; 团队文化; 组织学习

中文摘要. 近年来, 我国酒店迅猛发展的同时也迎来了越来越多的挑战, 如何提高入住率, 如 何将有下滑势头的酒店利润提升上来，如何满足消费者的多样化需求，成为各类型酒店管理 者的思考的首要问题。服务创新因其对提升酒店服务质量进而提高顾客满意度以及酒店利润 而受到酒店管理者以及学术研究者的重视。本文基于之前学者对服务创新的研究, 将从团队 文化和组织学习这两个角度探究其对酒店服务创新的作用, 以全国范围内主要是国内几大城 市的中高档酒店包括近年来突起的经济型酒店和主题酒店为研究对象, 分析现有酒店对服务 创新的重视程度以及是如何实现服务创新, 继而验证得出影响这些酒店服务创新的因素。

\section{1. 引言}

从目前我国企业创新管理的实践看，服务创新受到了前所未有的重视，这与目前的创新 大环境是分不开的。新世纪的到来, 也是互联网在我国普及的时代, 也是知识经济的时代。 我国经济深受互联网的影响, 市场竞争越来越激烈, 技术的引进很快会被同行业所模仿, 我 
们唯有不断创新才能保持企业的一直生存下去。我国酒店企业目前不仅面临国内市场的竞争, 同时也受国际品牌的如北美、欧洲等国际酒店品牌入驻中国的威胁，使得我国酒店业整体盈 利形式相较以前有很大的下滑。所以, 酒店企业要想保持竞争地位不仅与国内同行竞争更要 放眼全球, 就要进行市场细分, 做好市场定位针对其面临的目标顾客群体, 依其需求进行产 品创新; 同时还要比竞争对手更高的效率来吸引目标市场, 时时接受顾客反馈, 感知市场变 化, 提升企业市场竞争力。现有很多企业都明白服务创新的重要性, 但是却不知如何在提升 自己企业的服务创新能力, 而本文可以为酒店企业提供一个清晰的指引。所以, 本研究成果 可为我国酒店企业进行服务创新活动提供一些指导和借鉴, 提高其服务创新能力, 进而提升 竞争力。

\section{2. 研究现状}

\section{1 创新与服务创新概念的提出与演化}

“创新” 一词我们并不陌生, 虽然它没有很悠久的发展历史, 但是这个世纪它已经成为 各行各业都逃离不开的生存法宝。创新一词起源于拉丁语。它原有三层含义：1、更新；2、 创造新的东西; 3、改变。而作为一种理论创新概念最初是由美籍奥地利经济学家约瑟夫 - 熊 彼特 (J. A. Schumpeter) 在其 1911 年出版的德文版《经济发展理论》中提出的, 此书在 1934 年被译成英文时，使用了 “创新”（innovation）一词。

一般认为，服务与产品（有形）是不同的，服务的 “无形性”、“即时生产即时消费”、 “易逝性” 和 “不可储存性” 的特点决定了差别之所在（赖然, 2014） ${ }^{[1]}$ 。这种差异化的存 在, 也将现有研究文献划分为了两类: 服务创新与服务业的创新, 国外学者基于服务创新与 有形的产品创新的差异化的视角, 提出了服务创新的分类。我国国内服务创新国内服务创新 研究始于年代末, 目前仍处于起步阶段。关于服务创新分类研究的文献不多, 其中比较显著 的是魏江等人 $(2008,2009)$ 的分类, 他们在案例研究的基础上, 将服务创新分为概念创新与 传递创新 ${ }^{[2,3]}$ 。

\section{2 国内外酒店服务创新的相关研究}

在过去的二十年里, 世界经济重点从商品生产到服务的一个稳定的转变, 从而服务经济 占据着主导地位, 为了探索员工创新行为, Scott 和Bruce (1994) 通过访谈企业高层管理人 员开发出员工服务创新行为 (ESIB) 量表 ${ }^{[4]}$ 。员工将其创造性思维付诸实践转化成创新性行 为, 能为企业带来更新更好的创意、产品、服务和生产流程, 是企业实现突破性发展并取得 成功的有效途径 (刘泓, 魏文斌, 2009) ${ }^{[5]}$ 。 Meng-Lei Monica Hu, (2009) 的研究中指出 酒店服务创新表现在两个方面: 新服务开发 (NSD) 与员工服务创新 (ESIB) ${ }^{[6]}$ 。

\section{3. 研究假设与理论模型}

\section{1 组织学习对酒店服务创新的影响}

组织是一个不断创造知识的实体, 组织学习是指组织知识基础的形成和发展过程, 它也 是组织创造、获得和转移知识的过程。随着学习过程的进行, 新的知识不断地产生、积累和 有效运用, 从而推动了组织的知识创新。在新知识的开发中, 组织学习扮演了关键性的角色。 因此, 学习对于知识创新来说是必要的, 知识的扩展依赖于学习的强度以及先前的知识学习。 企业只有通过组织学习, 才能促进员工知识创新和发展核心能力, 并充分认识和利用外部环 境变化带来的发展机会。通过学习, 企业员工可以利用现存的知识生产出新知识。组织学习 是员工服务创新的基础与前提。因此, 本文提出以下研究假设:

H1a: 组织学习正向影响酒店企业员工服务创新。

H1b：组织学习正向影响酒店企业新服务开发。 


\section{2 团队文化对酒店服务创新的影响}

因为创新任务需要专业的知识在多方面的渗入，团队的发展就日益壮大。Quinn(1992) 的 84 例创新研究产生了七个促进组织创新重要的因素, 良好的团队精神是其中之一 ${ }^{[7] 。 才 队 ~}$ 成员之间的相互作用能防止误解, 增强价值观, 领导力, 信任和正义的分享, 从而促进创新 发挥更大的绩效。在国外的一些研究中他们将团队支持, 团队协作，团队凝聚力视为一些非 常重要的团队文化的因子。团队文化与服务创新绩效之间存在显著的正相关关系主要是因为 团队支持，协调和凝聚力文化可以激发团队的服务创新绩效。因此我们提出以下假设:

$\mathrm{H} 2 \mathrm{a}$ ：团队支持正向影响酒店企业员工服务创新绩效。

$\mathrm{H} 2 \mathrm{~b}$ ：团队支持正向影响酒店企业新服务开发。

$\mathrm{H} 2 \mathrm{c}$ ：团队协作正向影响酒店企业员工服务创新绩效。

$\mathrm{H} 2 \mathrm{~d}$ ：团队协作正向影响酒店企业新服务开发。

$\mathrm{H} 2 \mathrm{e}$ ：团队凝聚力正向影响酒店企业员工服务创新绩效。

H2f：团队凝聚力正向影响酒店企业新服务开发。

\section{3 模型框架}

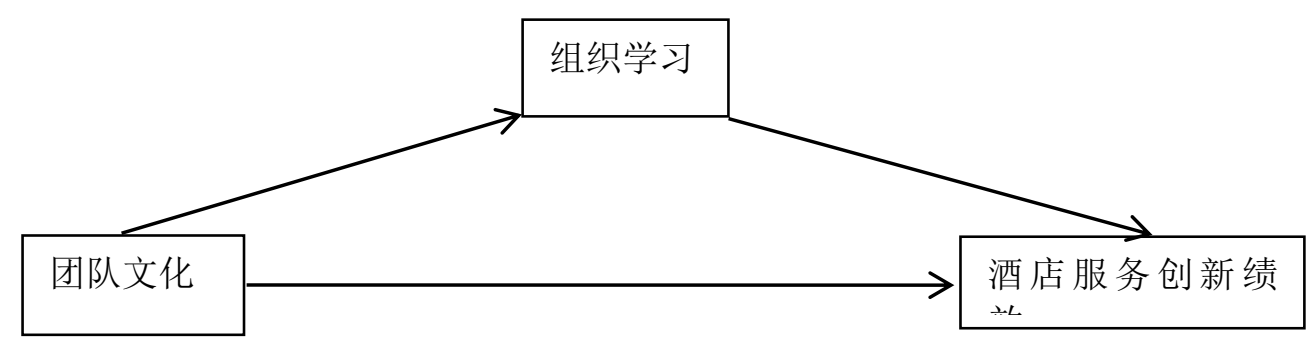

\section{4. 数据分析}

\section{1 变量的操作性定义与衡量}

为了更科学地对各变量进行定义, 并且较为权威地划分和测量各变量的维度, 本研究最大程 度地参考国内外权威的研究文献所采用的量表、问卷, 再结合本研究所在的具体的情景对问 卷加以适度的修改和完善。本研究的调查问卷共由两大部分组成, 前一部分是填写调查问卷 者的个人基本信息以及所工作酒店的基本资料; 第二大部分是本论文各变量的问题项本研究 调查问卷的题项选择七点量表制成, 问题项除了员工所在酒店部门这一题项是填写问卷者自 己填写文字答案 (不同酒店对相同部门的名称定义是不完全相同的, 为避免填写者迷惑不知 如何选择, 所以此题设计为调查问卷填写者自己写出所在酒店的部门) 之外皆为单选题, 答 案内容分为完全不符合、比较不符合、有点不符合、中立、有点符合、比较符合、完全符合 等, 由受测者勾选其一选项, 分数越高的问题项, 代表受测者对此问题项的同意程度越高。

综合国内外学者对组织学习以及组织学习与服务创新绩效、知识共享等的研究, 结合本 研究的实际情况, 主要参考了 Monica Hu (2009) ${ }^{[6]}$ 和 Dibella, A. J., Nevis, E. C (1996) ${ }^{[8]}$ 的相关研究, 本研究对组织学习的度量涉及 4 个问题项。研究指标、问题项和问卷来源论 述见表 4-2.

表 4-2 组织学习构面的衡量题项

\begin{tabular}{|c|c|c|}
\hline 指标 & 问题项 & 来源 \\
\hline \multirow{4}{*}{ 组织学习 } & Q1. 1 公司经常为员工组织培训, 提高我们的知识层面 & \multirow{4}{*}{$\begin{array}{l}\text { Monica Hu } \\
\text { (2009), } \\
\text { Dibella, } \\
\text { A. J., } \\
\text { Nevis, E. } \\
\text { C (1996) }\end{array}$} \\
\hline & Q1. 2 我想成为一名在同事眼中有专业知识的人 & \\
\hline & Q1. 3 帮助解决工作中的问题会让我感到快乐和满足 & \\
\hline & $\begin{array}{l}\text { Q1. } 4 \text { 公司良好的学习氛围更加促进我跟同事一起学习 } \\
\text { 和分享知识的欲望。 }\end{array}$ & \\
\hline
\end{tabular}


学术界对于团队文化的测量说法较多, 不同的学者从不同的角度看待团队文化, 对其进 行了度量, 但一直没有统一的测量维度, 本文量表主要来源于 Monica Hu (2009) 有关服务 创新绩效影响因素的研究 ${ }^{[6]}$ 。该量表是在对国外酒店广泛调研的基础上, 将反应团队文化因 素的维度（团队支持、团队协作、团队凝聚力）都包含在内, 能够比较全面的反应团队文化 的内涵。本文又考虑到国内外文化因素不同对本研究的影响, 在原量表的基础之上删除去与 中国国情不是很符合的几个题项，经过反复的推敲和预调研，形成本量表，包括团队支持、 团队协作、团队凝聚力三个维度，研究指标、问题和问卷来源论述见表 4-3。

表 4-3 团队文化构面的衡量题项

\begin{tabular}{|c|c|c|}
\hline 指标 & 问题项 & 来源 \\
\hline \multirow{3}{*}{ 团队支持 } & Q2. 1 我的领导支持我们共享知识和技术信息 & \multirow{10}{*}{$\begin{array}{l}\text { Monica Hu } \\
\quad(2009)\end{array}$} \\
\hline & $\begin{array}{l}\text { Q2.2 在我的工作中, 遇到困难时同事或领导会帮助 } \\
\text { 解决难题 }\end{array}$ & \\
\hline & Q2. 3 我以及身边的同事对知识和技术共享是支持的 & \\
\hline \multirow[t]{4}{*}{ 团队协作 } & $\begin{array}{l}\text { Q2. } 4 \text { 我所在的酒店在招聘人才的时候很重视团队合 } \\
\text { 作 }\end{array}$ & \\
\hline & Q2. 5 本酒店有正式的规则和程序来协调团队合作 & \\
\hline & Q2.6 本酒店领导具备协调团队合作的能力 & \\
\hline & Q2. 7 在业余时间, 酒店的团队成经常进行小型聚会 & \\
\hline \multirow{3}{*}{$\begin{array}{c}\text { 团队凝聚 } \\
\text { 力 }\end{array}$} & Q2. 8 我们团队拥有积极向上优秀的团队精神 & \\
\hline & Q2.9 我在团队工作中的参与感很强 & \\
\hline & $\begin{array}{l}\text { Q2. } 10 \text { 我们团队中的每一位成员都有助于酒店提高服 } \\
\text { 务质量 }\end{array}$ & \\
\hline
\end{tabular}

综合国内外学者对服务创新绩效的研究, 结合本研究的实际情况, 本研究在服务创新绩 效的维度划分中, 重点参考了Monica Hu（2009）的研究成果, 并将服务创新绩效分为员工 服务创新和新服务开发两个维度 ${ }^{[6]}$ 。如表 4-4 显示，本部分问卷一共有 10 个题目，其中， 1-6 题是对员工服务创新的衡量题目，7-10 题是对新服务开发的衡量题目。

表 4-4 服务创新绩效构面的衡量题项

\begin{tabular}{|c|c|c|}
\hline 指标 & $\begin{array}{l}\text { 问题项 } \\
\end{array}$ & 来源 \\
\hline \multirow{6}{*}{$\begin{array}{l}\text { 员工服 } \\
\text { 务创新 }\end{array}$} & Q3. 1 在工作中, 我对新的服务的提出或实施作出了贡献 & \multirow{10}{*}{$\begin{array}{c}\text { Monica } \\
\text { Hu } \\
\text { ( } 2009\end{array}$} \\
\hline & Q3. 2 在工作中, 我试着提出自己的服务创意并说服别人 & \\
\hline & Q3. 3 在工作中，我善于发现新的服务技术和方法 & \\
\hline & $\begin{array}{l}\text { Q3. } 4 \text { 在工作中, 我提供能够提高服务质量、令顾客满意的计 } \\
\text { 划 }\end{array}$ & \\
\hline & Q3. 5 在工作中, 我努力争取实现创新所需的资金和资源 & \\
\hline & $\begin{array}{l}\text { Q3. } 6 \text { 总的来说, 我认为自己是团队中的一员, 并认真做好自 } \\
\text { 己的工作 }\end{array}$ & \\
\hline \multirow{4}{*}{ 新服务 } & $\begin{array}{l}\text { Q3. } 7 \text { 我所在的酒店经常会发现一些新的资源, 适应时代的发 } \\
\text { 展 }\end{array}$ & \\
\hline & $\begin{array}{l}\text { Q3. } 8 \text { 本酒店现有的人力、物力资源, 有能力实现新服务开发 } \\
\text { 的需要 }\end{array}$ & \\
\hline & Q3. 9 在发展新业务时, 所有部门都能够有效地进行互动 & \\
\hline & $\begin{array}{l}\text { Q3. } 10 \text { 在开发新的服务项目时, 管理人员和一线服务人员可以 } \\
\text { 进行密切的合作 }\end{array}$ & \\
\hline
\end{tabular}




\section{2 样本选择与数据收集}

本研究为了更好地梳理清楚市场导向、团队文化、组织学习与服务创新绩效之间的关系， 通过对酒店企业员工进行调查分析, 挖掘问卷数据背后的真实原因, 为我们的实践工作做好 指导。考虑到样本的代表性和取样的便捷性, 本文主要选取华南地区（以广州为主）、华中 地区（以武汉为主）、华北地区（以北京为主）、华东地区（以上海为主）的酒店企业员工 作为研究对象。

\section{3 验证性因子分析}

根据前人的实证研究经验, 本研究认为, 适配度指标的理想数值范围是 GFI、CFI 均大 于 0.9 , 在 0.8-0.9 之间也可以接受; RMR 越小越好; RMSEA 的值应该低于 0.05 , 在 0.05-0.08 之间也可以接受。本研究验证性因素分析的各项指标如下表 4-20 所示, 除了 “团 队文化” 的 RMSEA 指数略大于 0.08 之外, 其他指标均在可接受的范围之内, 表明本问卷具 有良好的结构效度。

表 4-20 验证性因子分析结果

\begin{tabular}{c|c|c|c|c|c|c|c}
\hline 拟合指数 & $x^{2} / \mathrm{df}$ & GFI & CFI & TLI & RMR & RMSEA & NFI \\
\hline 市场导向 & 2.266 & 0.949 & 0.969 & 0.958 & 0.083 & 0.065 & 0.946 \\
\hline 团队文化 & 2.935 & 0.938 & 0.971 & 0.959 & 0.057 & 0.082 & 0.957 \\
\hline 组织学习 & 6.518 & 0.980 & 0.984 & 0.952 & 0.039 & 0.136 & 0.981 \\
\hline $\begin{array}{c}\text { 服务创新绩 } \\
\text { 效 }\end{array}$ & 3.679 & 0.925 & 0.956 & 0.941 & 0.071 & 0.095 & 0.941 \\
\hline
\end{tabular}

\section{4 结构方程模型拟合}

模型的标准化回归路径系数

\begin{tabular}{|c|c|c|c|c|c|}
\hline 路径 & 估计值 & 标准值 & SE & C. $R$ & $\mathrm{P}$ \\
\hline 组织学习<---团队支持 & .895 & .832 & .189 & 11.336 & $* * *$ \\
\hline 组织学习<---团队协作 & -1.465 & -1.362 & .255 & -0.002 & 0.999 \\
\hline 组织学习<---团队凝聚力 & .952 & .885 & .078 & 7.541 & $* * *$ \\
\hline 员工服务创新<---团队支持 & .601 & .481 & .138 & 3.256 & $* * *$ \\
\hline 新服务开发〈---团队支持 & .135 & .108 & .036 & 1.982 & $* * *$ \\
\hline 员工服务创新 $<---$ 团队协作 & .374 & .299 & .018 & 2.317 & $* * *$ \\
\hline 新服务开发〈---团队协作 & .390 & .312 & .071 & 2.729 & $* * *$ \\
\hline $\begin{array}{c}\text { 员工服务创新 }<--- \text { 团队凝聚 } \\
\text { 力 }\end{array}$ & .153 & .212 & .087 & 2. 002 & $* * *$ \\
\hline 新服务开发〈---团队凝聚力 & .010 & .014 & .008 & 0.843 & 0.05 \\
\hline 员工服务创新 $<---$ 组织学习 & 1.098 & 0.891 & .167 & 17.104 & $* *$ \\
\hline 新服务开发<---组织学习 & .289 & 0.401 & .056 & 2.583 & $* * *$ \\
\hline
\end{tabular}

\section{5 假设检验}

在整合模型检验中，组织学习与酒店企业员工服务创新的正相关关系通过显著性检验， 标准化路径值为 0.891 , 组织学习与酒店企业新服务开发的正相关关系通过显著性检验, 标 准化路径值为 0.401 , 因此, 假设 $\mathrm{H} 1 \mathrm{a}$ 和假设 $\mathrm{H} 1 \mathrm{~b}$ 得到支持。

团队支持与酒店企业员工服务创新的正相关关系通过显著性检验，标准化路径值为 0.481, 团队支持与酒店企业新服务开发的正相关关系通过显著性检验, 标准化路径值为 0.108 , 团队协作与酒店企业员工服务创新的正相关关系通过显著性检验, 标准化路径值为 0.299 , 团队协作与酒店企业新服务开发的正相关关系通过显著性检验, 标准化路径值为 
0.312, 团队凝聚力与酒店企业员工服务创新的正相关关系通过显著性检验, 标准化路径值为 0.212 , 团队凝聚力与酒店企业新服务开发的相关关系未通过显著性检验, 标准化路径值为 0. $014 （ \mathrm{P}=0.05 ）$ ，所以，假设 $\mathrm{H} 2 \mathrm{a} 、 \mathrm{H} 2 \mathrm{~b} 、 \mathrm{H} 2 \mathrm{c} 、 \mathrm{H} 2 \mathrm{~d} 、 \mathrm{H} 2 \mathrm{e}$ 得到支持，假设 $\mathrm{H} 2 \mathrm{f}$ 不支持。

\section{5. 结论}

\section{1 团队文化对酒店服务创新绩效的影响}

到目前为止, 只有少量的实证研究在本领域进行了探讨, 过去的大多数研究重点集中在 个别员工创新的前因上。但是, 团队越来越多地承担起组织服务创新的任务。本文检验了酒 店服务创新和酒店团队的团队文化之间的积极关系。实证数据支持 5 个假设, 表明团队支持 和酒店服务创新、团队协作和酒店服务创新、团队凝聚力和酒店服务创新都有非常重要的直 接关系。

团队文化与酒店服务创新绩效的影响关系中本文在理论基础上设置了 6 个假设, 团队文 化（团队支持、团队协作、团队凝聚力）与酒店员工服务创新和团队协作、团队支持与新服 务开发等五个假设通过显著性检验, 这一结果关于团队协作、团队凝聚力与员工服务创新显 著正相关与颜宁 (2011) ${ }^{[9]}$ 和 Monica Hu 等 (2009) ${ }^{[6]}$ 等的结论较为一致。

优秀的团队文化在促进酒店服务创新方面扮演着非常重要的角色。团队支持和团队协作、 团队凝聚力对于服务创新的积极作用是非常显著的。从估计系数得出了一个结论, 有高度团 队凝聚力和团队协作的酒店员工往往会有更多的服务创新行为, 同时高度的团队协作和支持 让员工更容易开发新服务。最后, 还没有足够的根据去证实团队凝聚力和新服务开发之间的 直接关联。

因此, 应该培养良好的团队文化, 促进酒店企业更好的内部交流环境。在服务型的企业 里: 要想有高度服务创新行为, 酒店组织首先要开发和确保团队协作和相互支持行为的实现; 另外, 良好的团队文化也能促进服务创新行为的实现。因为一个基于合作、组织支持和有凝 聚力的团队文化将能够更好地刺激组织内的知识共享, 同时也能够刺激和提高组织内个人创 新的积极性。

\section{2 组织学习对酒店服务创新绩效的影响}

根据模型验证结果可知, 组织学习对酒店企业的服务创新绩效的两个维度 (员工服务创 新和新服务开发) 均产生积极的正向影响作用。国外大量的组织学习的文献中, 学者所关注 的焦点大多围绕着组织学习本身和对促进组织学习过程的组织环境或条件来进行研究和探 讨。

本文的研究题量关于组织学习的设计上同时考虑到了组织学习本身和组织促进组织学习 过程的组织环境这两点, 酒店企业员工的服务创新需要建立在组织学习的基础上对环境也有 很高的要求, 所以要想提高酒店服务创新的能力, 企业需要更加重视给员工提供有利的促进 组织学习的环境或条件, 让潜在需求、想法, 能够发展成为成熟的服务创新行为并加以实施。 另一方面, 与员工服务创新不同, 酒店新服务的发展则是需要在有利的环境中, 更需要组织 学习本身, 我们需要可以在无形的服务的基础之上, 进行改造和创造的能力, 而这种能力的 获取最直接有效的途径就是学习, 所以, 酒店企业也要注重对企业员工组织学习的安排与计 划。

\section{3 管理启示}

酒店企业要实现服务创新, 要注重培养团队文化, 善于挖掘员工的创造性思维, 积极激 发员工的服务创新行为。根据本文的研究结论, 企业可以主要从组织学习、团队文化这两方 面进行服务创新管理机制的设计。

5.3. 1 营造有利于员工创新行为的团队文化

服务企业要敢于承担创新风险, 支持创新行为。员工往往都认为创新意味着容易错误和 
失败, 这会限制服务企业员工的创新行为。因此, 作为一个企业的领导者, 应该明确支持创 新, 允许员工去实现他们的新想法, 并且能够设立专门的创新团队帮助创新想法的提供者把 初步想法转化为实际可操作的服务行为。

5. 3.2 制定长期有利于新服务开发的创新流程

通过此次调查研究发现, 酒店企业从管理者到基础员工都了解新服务开发对于企业生存 发展的重要性, 但能坚持为新服务开发尽可能的创造有利条件的酒店企业却不多, 究其原因, 新服务的开发需要长期的投入才会产生直接的财务绩效, 这会使得多数酒店企业在中途将资 金投入到其他部门。所以, 酒店企业应该制定出一个能够长期坚持的创新流程, 来支持企业 的新服务开发。

5.3 .3 建立有利于员工参与服务创新的体制

从组织结构上看, 可以调整现有的组织结构, 进行组织结构的创新, 建立专门的服务创 新管理部门（岗位），让员工知道发现创新机会后如何利用企业资源进行创新构想的调查、 研究、拥护以及应用, 让员工不仅仅是创新思想的来源更能为服务创新的调查、研究、执行 作出贡献。其次, 可以在绩效考核中增添创新能力考评一项, 分等级进行服务创新奖励, 不 能因为创新提案最终没有实施就否定整个过程所付出的努力, 打击员工为企业提供创新来源 的积极性。

\section{References}

[1] Lai ran, Xu Ming,Study on the development of international service innovation and the visualization of the research frontier[J],Forward Position, vol. 19, pp.21-23,2013

[2] Wei Jiang, Jiao Hao. Research on the relationship among Entrepreneurial Orientation, organizational learning and dynamic capabilities[J]. Foreign Economics \& Management,vol.02,PP.36-41,2008

[3] Wei Jiang, Wang Tongan, Lu Ping. An Empirical Study on the relationship between innovation organization structure and innovation performance of knowledge intensive service enterprises[J]. Journal of Industrial Engineering andEngineering Management,vol.03,PP.103-110,2009

[4] Scott S C. \& Bruce R A .Determinates of innovative behavior: A path model of individual innovation in the work-place[J]. Academy of Management journal, vol.37, PP.580-607, 1994

[5] Liu Hong, Wei Wenbin, The influence of enterprise innovation atmosphere on employees' creativity and the measures[J],Management and Administration, PP.12-13,2009

[6] Meng-Lei Monica Hu. Knowledge Sharing and innovative service behavior relationship : guanxi as mediator.[J].Social Behavior and Personality, Vol.37, PP.977-992,2009

[7] Quinn, J. B ,"Innovation and Corporate Strategy: Managed Chaos", in Technology in the Modern Corporation: A Strategic Perspective, Mel Horwich, ed, New York: Pergamon Press., 1986

[8] Dibella, A. J., Nevis, E. C., \& Gould, J. M. Understanding organizational learning capability [J]. Journal of Management Studies, 1996;33, 361-379.Senge, P.. The fifth discipline. NY: Doubleday.1990

[9] Yan Ning. Research on the relationship among team culture, knowledge sharing and service innovation: a case study of Fujian high star hotels[J]. Journal of Beijing International Studies University,vol.03,pp.56-64+55,2011 